\title{
\begin{tabular}{l|l} 
Mibraries & DSpace@MIT
\end{tabular}
}

\author{
MIT Open Access Articles
}

\begin{abstract}
An integrated modeling framework for infrastructure system-of-systems simulation
\end{abstract}

The MIT Faculty has made this article openly available. Please share how this access benefits you. Your story matters.

Citation: Grogan, Paul T., and Olivier L. de Weck. "An integrated modeling framework for infrastructure system-of-systems simulation." In 2013 IEEE International Systems Conference (SysCon), 483-490. Institute of Electrical and Electronics Engineers, 2013.

As Published: http://dx.doi.org/10.1109/SysCon.2013.6549926

Publisher: Institute of Electrical and Electronics Engineers (IEEE)

Persistent URL: http://hdl.handle.net/1721.1/84056

Version: Author's final manuscript: final author's manuscript post peer review, without publisher's formatting or copy editing

Terms of use: Creative Commons Attribution-Noncommercial-Share Alike 3.0 


\section{An Integrated Modeling Framework for Infrastructure System-of-systems Simulation}

\author{
Paul T. Grogan \\ Engineering Systems Division \\ Massachusetts Institute of Technology \\ Cambridge, Massachusetts, USA \\ Email: ptgrogan@mit.edu
}

\author{
Olivier L. de Weck \\ Engineering Systems Division and \\ Department of Aeronautics and Astronautics \\ Massachusetts Institute of Technology \\ Cambridge, Massachusetts, USA \\ Email: deweck@mit.edu
}

\begin{abstract}
Design of future hard infrastructure must consider emergent behaviors from cross-system interdependencies. Understanding these interdependencies is challenging due to high levels of integration in high-performance systems and their operation as a collaborative system-of-systems managed by multiple organizations. Existing modeling frameworks have limitations for strategic planning either because important spatial structure attributes have been abstracted out or behavioral models are oriented to shorter-term analysis with a static network structure. This paper presents a formal modeling framework as a first step to integrating infrastructure system models in a system-of-systems simulation addressing these concerns. First, a graph-theoretic structural framework captures the spatial dimension of physical infrastructure. An element's simulation state includes location, parent, resource contents, and operational state properties. Second, a functional behavioral framework captures the temporal dimension of infrastructure operations at a level suitable for strategic analysis. Resource behaviors determine the flow of resources into or out of nodes and element behaviors modify other state including the network structure. Two application use cases illustrate the usefulness of the modeling framework in varying contexts. The first case applies the framework to future space exploration infrastructure with an emphasis on mobile system elements and discrete resource flows. The second case applies the framework to infrastructure investment in Saudi Arabia with an emphasis on immobile system elements aggregated at the city level and continuous resource flows. Finally, conclusions present future work planned for implementing the framework in a simulation software tool.
\end{abstract}

\section{INTRODUCTION}

Modern societies rely on widespread infrastructure to provide critical services which in turn enable productive societal activities. The U.S. Department of Homeland Security identifies 18 critical infrastructure sectors, of which we focus on a subset relating to physical resource management such as agriculture and food, energy, water and wastewater, communications, and transportation [1]. These "hard" infrastructure sectors consist of large physical networks of interrelated components which produce and transport resources. Like other engineering systems, they are large-scale, high-cost, and longliving, motivating strategic decisions for their design and operation to maximize life-cycle value. With an emphasis on sustainability, life-cycle value can be decomposed to economically efficient performance, environmentally responsible impacts, and social equity of access to services [2].
Infrastructure are intricately woven of technical components having coupled structure and behaviors. System-level emergence contributes to cases where individual events yield unexpected, widespread behaviors across system boundaries. A few examples include the 1994 Northridge earthquake, September 2001 World Trade Center disaster, and August 2003 Northeast blackout [3], [4], [5]. System coupling in these cases arises from various types of interdependencies across systems: physical resource flows, cyber information flows, geographic co-location, or other logical dependencies [6].

To emphasize the impact of infrastructure interdependencies on performance, consider complexity as "a measure of uncertainty in achieving the specified [functional requirements]," here, life-cycle value or sustainability objectives [7]. Sources of complexity in infrastructure systems include structurecomponents (system elements), interactions between elements, and the system architecture-and behavior over short and long time-scales [8]. These sources of complexity are illustrated in Fig. 1 for a notional infrastructure system. Reference [9] quantifies architectural complexity as a function of the graph energy of system dependency network. As a measure of entropy, graph energy is lowest for centralized architectures and increases for more distributed architectures. Infrastructure systems having more interconnected components in a distributed structure exhibit higher architectural complexity which is linked to challenges of system integration.

In the face of higher complexity, some hard infrastructure seek to strengthen system coupling for improved performance (e.g. resource efficiency) through element-element, elementsystem, and system-system integration. ${ }^{1}$ Element-element integration combines the functions of coupled infrastructure elements in an integrated element. For example, cogeneration and combined cycle plants use waste heat from electricity generation for a secondary purpose such as heating or water desalination. Element-system integration combines the functions of coupled infrastructure elements with an existing system. For example, future electric vehicles may interconnect with the electrical grid to provide energy storage for variable renew-

\footnotetext{
${ }^{1}$ There is a subtle distinction between elements and systems specified by an implicit unit of analysis. In general, elements are the lowest-level unit implemented in a model which are aggregated into systems; however one model's elements may be another's systems.
} 


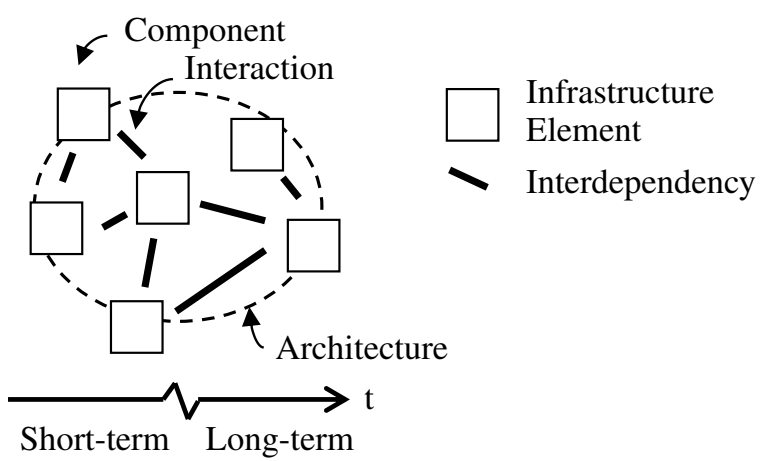

Fig. 1. A notional infrastructure system represented as a graph of elements and their interdependencies highlighting component, interaction, and architectural sources of structural complexity and short- and long-term behavioral complexity.

able sources such as solar and wind. Finally, system-system integration combines coupled systems in a directed system-ofsystems built and centrally managed to fulfill specific purposes [10]. For example, the International Space Station integrates multiple systems such as atmospheric control, water recovery, thermal regulation, and waste handling to sustain crew life in a partially closed-loop environment. Larger-scale terrestrial infrastructure systems such as Masdar City in Abu Dhabi, conceptualized as a carbon-neutral, zero waste city, will also require integration of transportation, electricity, and building systems to meet sustainability goals [11].

The lack of a single central authority in a directed systemof-systems is a barrier to widespread system-system integration. Infrastructure systems are often independently managed by multiple public agencies and commercial firms and operate more like a collaborative system-of-systems [10]. No one organization has complete control and some level of collaborative design is required to achieve mutual gains. This point emphasized in a recent National Research Council report which warns that "focusing on one system, one issue, or one problem at a time, the nation runs the risk of wasting increasingly scarce resources and creating new problems for future generations," calling for "collaborative, systems-based approaches to leverage available resources and provide for cost-effective solutions across institutional and jurisdictional boundaries" [12]. In the absence of a central authority, the architectural complexity, i.e. integration of component systems, is a significant challenge to implementing infrastructure systems.

Thus, a tension emerges in modern infrastructure design: a desire for increased performance to meet sustainability goals seeks to increase integration; however the resulting complexity impedes understanding of the system behaviors and may contribute to overall lower performance. To address this issue, this paper develops a common modeling framework for future analysis of infrastructure system-of-systems in an integrated simulation environment. The framework considers both structure and behavior as sources of complexity with an emphasis on system architecture to enable integration of infrastructure models. The modeling framework, once implemented in a simulation model, could contribute to improved complex infrastructure system design.

This paper is organized as follows. Section II provides an overview of past efforts in integrated modeling and simulation for infrastructure systems. Section III presents the formal modeling framework which captures structural and behavioral dimensions of infrastructure system-of-systems. Section IV applies the modeling framework to two use cases to verify its generality and usefulness. Section V concludes with future work for simulation implementations.

\section{LITERATURE REVIEW}

A large body of literature over the past decade addresses challenges associated with understanding infrastructure systems. Many efforts are related to security and protection planning under the U.S. Department of Homeland Security and other national infrastructure protection programs. Reference [3] surveys interdependency modeling in critical infrastructure. The brief overview provided here identifies three areas of contributing literature: conceptual frameworks, high-level aggregate models of system behaviors, and operational system reliability models.

The first area of literature includes conceptual frameworks of constructs which help create a common understanding of factors relating to infrastructure systems. However, conceptual frameworks are not executable or computable as a simulation model and only guide this research effort. Reference [6] identifies physical, cyber, geographic, and logical categories of infrastructure interdependencies. From this perspective, interdependencies are static attributes of the built system structure, not necessarily behaviors related to nominal operation. Further describing behaviors, [13] presents a functional classification for complex systems consisting of a $5 \times 5$ matrix of operands and operations shown in Table I. Systems are classified by one or more functions-for example, a power plant is $F_{M} / F_{E}$ as it transforms matter to create energy.

A second area of literature uses aggregated infrastructure simulation models for high-level understanding of interdependencies. Several examples use the system dynamics formalism to specify the behavior of coupled infrastructure [14], [15]. These approaches use aggregated system models with explicit couplings specified by shared state variables. Some applications also consider soft infrastructure or social aspects of the technical systems. The system dynamics formalism allows for dynamic response to disturbances, however the data are aggregated at the system level such that structural changes from planning or design activities for specific infrastructure elements are not considered separately. For example, resources such as water have transportation costs proportional to spatial attributes such as elevation change and distance traveled which are commonly abstracted out of the aggregated system model.

A third area of literature uses detailed simulation models to represent infrastructure operations with the objective of analyzing short-term behaviors resulting from disturbances. To 
TABLE I

System FunCtion Classified By PRocess AND Five OPERANDS

\begin{tabular}{rccccc}
\hline Process & Organisms & Matter & Energy & Information & Currency \\
\hline Transform & $F_{O}$ & $F_{M}$ & $F_{E}$ & $F_{I}$ & $F_{C}$ \\
Transport & $P_{O}$ & $P_{M}$ & $P_{E}$ & $P_{I}$ & $P_{C}$ \\
Store & $S_{O}$ & $S_{M}$ & $S_{E}$ & $S_{I}$ & $S_{C}$ \\
Exchange & $X_{O}$ & $X_{M}$ & $X_{E}$ & $X_{I}$ & $X_{C}$ \\
Control & $C_{O}$ & $C_{M}$ & $C_{E}$ & $C_{I}$ & $C_{C}$ \\
\hline
\end{tabular}

increase modeling detail, this class of models use networkbased or graph-theoretic representations of infrastructure elements. For example, [16] define a node as "an entity that acts as a source, produces, consumes, or transforms a resource..." and an edge as "a physical or virtual entity that acts as a conduit for flow for a physical quantity, information, or influence ... [representing] a direct level of dependence." Here, physical, informational, geospatial, policy/procedural, and societal dependencies each have a coupled structure-behavior representation in the infrastructure network. Another approach uses a multi-layered network flow formulation to represent interdependencies among infrastructure systems [17]. In this case. behaviors are determined in the context of an computable general equilibrium problem, operationalized by agent-based simulation methods. In both cases, infrastructure networks are assumed to remain static over the course of a simulation, a reasonable assumption for operational response. However, guiding strategic decisions of infrastructure design and planning happen over longer timescales and at higher levels of aggregation with dynamic infrastructure network structure and behavior.

To summarize, the existing literature includes conceptual frameworks for infrastructure structure and behavior, however these constructs alone are not executable as a model. High-level system dynamics models rely on interdependencies defined by shared variables and do not consider the spatial structure of infrastructure elements. On the other hand, flownetwork models representing both element-level structure and behavior operate on a short time-scale with a static network structure. Thus, the gap our proposed modeling framework seeks to fill is to:

1) Disaggregate infrastructure systems into component elements representing spatial structure

2) Aggregate infrastructure system behaviors at scales suitable for strategic planning

3) Allow for dynamic infrastructure network structure corresponding to design and planning activities

The next section describes a formal modeling framework addressing these objectives for application in a simulation tool supporting strategic infrastructure system-of-systems design.

\section{Formal Modeling Framework}

This section discusses the formal modeling framework in detail. First, the structural components are described as spatial simulation state. Second, the behavioral components are described as temporal simulation state changes.

\section{A. Spatial-Structural Framework}

The spatial-structural framework describes the instantaneous state of an infrastructure system-of-systems using formal definitions. It includes a context framework applicable across models in a common domain and an instantiation framework for the unique components in a particular model.

1) Context Framework: The context framework defines the allowable locations and resource types which can be used across multiple model instantiations. In practice, each context framework implementation is targeted for an application domain representing unique spatial characteristics.

Locations are based on the graph-theoretic concept of nodes, defined in this case as spatial units of aggregation where resources are transferable between co-located infrastructure. Some infrastructure are positioned between nodes as mobile (e.g. trucks, ships) or fixed (e.g. pipelines) distribution components. Thus, for a set of nodes $\mathbf{N}$, the set of allowable locations is a set of node pairs

$$
\mathbf{L}=\left\{l_{i}\right\}: l_{i}=\left(n_{0}, n_{1}\right)_{i}, n_{0}, n_{1} \in \mathbf{N} \forall i
$$

where $l_{i}$ is a nodal location if $n_{0}=n_{1}$ and an edge location if $n_{0} \neq n_{1}$. Edge locations are directed such that $n_{0}$ is the origin node and $n_{1}$ is the destination node. $\mathbf{L}$ may not be a complete graph if constraints prevent infrastructure between certain nodes (e.g. non-physically adjacent nodes for ground transportation).

The allowable resource types is a set

$$
\mathbf{T}=\left\{t_{i}\right\}
$$

where each resource type $t_{i}$ corresponds to a mass-based (e.g. water), energy-based (e.g. electrical energy), informationbased (e.g. bits), currency-based (e.g. US dollars) or any other resource measured using a ratio scale with a non-arbitrary zero point. A set of resources $r$ is realized as a set of pairs of a resource type and a positive real quantity, i.e.

$$
r=\left\{(t, q)_{i}\right\}: t \in \mathbf{T}, q \in \mathbb{R}^{+} \forall i .
$$

Resources are aggregated by resource type and are fungible by type, whereas infrastructure are uniquely enumerated as discussed in the next section.

2) Instantiation Framework: The instantiation framework describes the infrastructure elements participating in a systemof-systems. The instantiated elements are a set

$$
\mathbf{E}=\left\{e_{i j}\right\}
$$

where each element $e_{i j}$ is component $j$ of system $i$. In this formulation, each element is uniquely assigned to one system; however rather than sector-specific resource functionality, the system assignment designates management or control of infrastructure elements. For example, a combined-cycle power/desalination plant may operate with both water and electricity resources, but is only managed by one organization (which may differ from other water or energy infrastructure).

Elements have four stateful properties: resource contents, location, parent element, and operational state. Elements are 
the only containers of resources in the modeling framework. Resources contained within an element are identified by a contents function

$$
\mathcal{C}=\mathcal{C}(e): e \mapsto r
$$

which maps an element $e \in \mathbf{E}$ to a set of resources $r$.

Elements exist at only one location at a time. An element's spatial position is identified by the location function

$$
\mathcal{L}=\mathcal{L}(e): e \mapsto l \in \mathbf{L}
$$

which maps an element $e \in \mathbf{E}$ to an allowable location $l$. Shorthand notations $\mathcal{L}_{0}(e)$ and $\mathcal{L}_{1}(e)$ are used to identify the origin and destination nodes for directed elements.

In addition to spatial location, elements can also be arranged in a hierarchical structure of nested relationships. Nested structure is identified by the parent function

$$
\mathcal{P}=\mathcal{P}(e): e \mapsto e_{\text {parent }} \in \mathbf{E}
$$

which maps an element $e \in \mathbf{E}$ to the element containing it. An element not nested inside another element is defined to be its own parent, i.e. $\mathcal{P}(e)=e$. The parent function may also be raised to multiple powers to map an element to its $n$th parent, e.g. a "grandparent" relationship is $\mathcal{P}^{2}(e)=\mathcal{P}(\mathcal{P}(e))$.

Finally, any other attributes necessary to describe an element's state are defined in the state function

$$
\mathcal{S}=\mathcal{S}(e): e \mapsto s \in S_{e}
$$

which maps an element $e \in \mathbf{E}$ to a state $s$ among its set of allowable states $S_{e}$. In practice each operational state is linked to a behavioral model to express one or more behaviors described in the following section.

\section{B. Temporal-Behavioral Framework}

The temporal-behavioral framework describes state changes which take place during a simulation execution. Behaviors are categorized as operating either on resources or elements. The formulations described here assume that any required preconditions such as spatial compatibility or capacity constraints are satisfied. Any condition violations should generate errors in an implementation of the framework.

1) Resource Behaviors: Resource behaviors are exhibited by elements to produce, move, or consume resources during a simulation. The four resource behaviors include storing, transporting, transforming, and exchanging illustrated in Fig. 2 , though many elements will compose multiple behaviors in a single model.

All four resource behaviors affect the flow of resources within and between nodes. As the units of spatial aggregation, there must be zero net flow of resources across each node's control boundary such the total resource flow out of a node $\mathcal{F}_{\text {out }}$ equals the total resource flow into the node $\mathcal{F}_{\text {in }}$, as shown in (9).

$$
\mathcal{F}_{\text {in }}(n)=\mathcal{F}_{\text {out }}(n) \forall n \in \mathbf{N}
$$

The aggregation of resource flows at nodes allows for more loosely-coupled element model implementations. It sets the

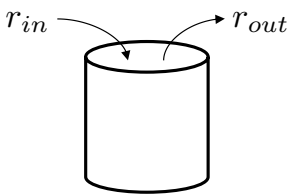

Storing

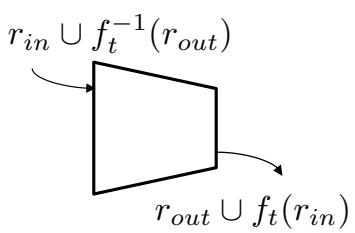

Transforming

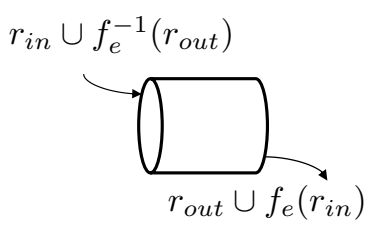

Transporting

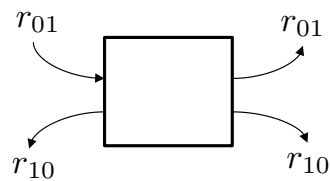

Exchanging
Fig. 2. Storing, transporting, transforming, and exchanging behaviors modify nodal resource flows by consuming and producing resources.

interface between systems at the nodal level which, in the limiting cases, may encompass all elements at one node (similar to a system dynamics model), or each element at a separate node (similar to a flow-network model).

The resource storing behavior captures the storage or retrieval of resources from an element's internal contents. It is a function of an element $e \in \mathbf{E}$, resources to store $r_{i n}$, and resources to retrieve $r_{\text {out }}$ with state changes specified in (10). ${ }^{2}$ Loss functions $f_{s}$ and $f_{r}$ describe inefficiencies during storage and retrieval actions respectively. For example, perfect storage would be defined by the identify function $f_{s}(r)=r$.

$$
\begin{gathered}
\mathcal{R}_{\text {store }}=\mathcal{R}_{\text {store }}\left(e, r_{\text {in }}, r_{\text {out }}\right) \\
\mathcal{C}(e) \leftarrow\left(\mathcal{C}(e) \cup f_{s}\left(r_{\text {in }}\right)\right) \backslash f_{r}\left(r_{\text {out }}\right) \\
\mathcal{F}_{\text {out }}\left(\mathcal{L}_{0}(e)\right) \leftarrow \mathcal{F}_{\text {out }}\left(\mathcal{L}_{0}(e)\right) \cup r_{\text {in }} \\
\mathcal{F}_{\text {in }}\left(\mathcal{L}_{1}(e)\right) \leftarrow \mathcal{F}_{\text {in }}\left(\mathcal{L}_{1}(e)\right) \cup r_{\text {out }}
\end{gathered}
$$

The resource transporting behavior describes movement of resources between nodes on an edge-located element. It is a function of an element $e \in \mathbf{E}$, input resources $r_{i n}$ for supply-driven "push" behavior, and output resources $r_{\text {out }}$ for demand-driven "pull" behavior with state changes shown in (11). Again, loss functions $f_{e}$ and $f_{e}^{-1}$ describe inefficiencies between specified input and output flow and between specified output and input flow respectively.

$$
\begin{aligned}
& \mathcal{R}_{\text {trsp }}=\mathcal{R}_{\text {trsp }}\left(e, r_{\text {in }}, r_{\text {out }}\right) \\
& \quad \mathcal{F}_{\text {out }}\left(\mathcal{L}_{0}(e)\right) \leftarrow \mathcal{F}_{\text {out }}\left(\mathcal{L}_{0}(e)\right) \cup r_{\text {in }} \cup f_{e}^{-1}\left(r_{\text {out }}\right) \\
& \mathcal{F}_{\text {in }}\left(\mathcal{L}_{1}(e)\right) \leftarrow \mathcal{F}_{\text {in }}\left(\mathcal{L}_{1}(e)\right) \cup r_{\text {out }} \cup f_{e}\left(r_{\text {in }}\right)
\end{aligned}
$$

The resource transforming behavior allows transformation between resource types. It is a function of an element $e \in \mathbf{E}$, input resources $r_{i n}$ for supply-driven behavior, and output resources $r_{\text {out }}$ for demand-driven behavior with state changes are specified in (12). Similar in form to the transporting behavior, it uses transformation functions $f_{t}$ and $f_{t}^{-1}$ to map

\footnotetext{
${ }^{2}$ A word on notation: the expression $\mathcal{X} \leftarrow \mathcal{X}^{\prime}$ used in this section indicates the value of a state expression $\mathcal{X}$ has been changed to $\mathcal{X}^{\prime}$ to exhibit a behavior.
} 
specified input to output resources and vice-versa.

$$
\begin{aligned}
& \mathcal{R}_{\text {trs } f}=\mathcal{R}_{\text {trsf }}\left(e, r_{\text {in }}, r_{\text {out }}\right) \\
& \quad \mathcal{F}_{\text {out }}\left(\mathcal{L}_{0}(e)\right) \leftarrow \mathcal{F}_{\text {out }}\left(\mathcal{L}_{0}(e)\right) \cup r_{\text {in }} \cup f_{t}^{-1}\left(r_{\text {out }}\right) \\
& \mathcal{F}_{\text {in }}\left(\mathcal{L}_{1}(e)\right) \leftarrow \mathcal{F}_{\text {in }}\left(\mathcal{L}_{1}(e)\right) \cup r_{\text {out }} \cup f_{t}\left(r_{\text {in }}\right)
\end{aligned}
$$

The resource exchanging behavior consists of a two-way directed flow of resources between elements. It is a function of two elements with paired origin-destinations, i.e. $e_{0}, e_{1} \in \mathbf{E}$ : $\mathcal{L}_{0}\left(e_{0}\right)=\mathcal{L}_{1}\left(e_{1}\right), \mathcal{L}_{1}\left(e_{0}\right)=\mathcal{L}_{0}\left(e_{1}\right)$, and two-way resources to exchange $r_{0,1}$ and $r_{1,0}$ with state changes shown in (13).

$$
\begin{gathered}
\mathcal{R}_{\text {xch }}=\mathcal{R}_{\text {xch }}\left(e_{0}, e_{1}, r_{0,1}, r_{1,0}\right) \\
\mathcal{F}_{\text {out }}\left(\mathcal{L}_{0}\left(e_{0}\right)\right) \leftarrow \mathcal{F}_{\text {out }}\left(\mathcal{L}_{0}\left(e_{0}\right)\right) \cup r_{0,1} \\
\mathcal{F}_{\text {in }}\left(\mathcal{L}_{1}\left(e_{0}\right)\right) \leftarrow \mathcal{F}_{\text {in }}\left(\mathcal{L}_{1}\left(e_{0}\right)\right) \cup r_{0,1} \\
\mathcal{F}_{\text {out }}\left(\mathcal{L}_{0}\left(e_{1}\right)\right) \leftarrow \mathcal{F}_{\text {out }}\left(\mathcal{L}_{0}\left(e_{1}\right)\right) \cup r_{1,0} \\
\mathcal{F}_{\text {in }}\left(\mathcal{L}_{1}\left(e_{1}\right)\right) \leftarrow \mathcal{F}_{\text {out }}\left(\mathcal{L}_{1}\left(e_{1}\right)\right) \cup r_{1,0}
\end{gathered}
$$

The resource exchanging behavior does not include a loss or transformation function, making it especially useful as the interface between independent infrastructure systems.

2) Element Behaviors: Element behaviors are exhibited by elements to change state attribute values other than resource contents, allowing for dynamic network structure. The three element behaviors include storing, transforming, and transporting.

The element storing behavior allows elements to be stored as cargo inside other elements. It is a function of two co-located elements, $e_{0}, e_{1} \in \mathbf{E}: \mathcal{L}\left(e_{0}\right)=\mathcal{L}\left(e_{1}\right)$ with state changes shown in (14).

$$
\begin{gathered}
\mathcal{E}_{\text {store }}=\mathcal{E}_{\text {store }}\left(e_{0}, e_{1}\right) \\
\mathcal{P}\left(e_{0}\right) \leftarrow e_{1}
\end{gathered}
$$

The element transforming behavior allows elements to change operational parameters. It is a function of an element $e \in \mathbf{E}$ and one of its allowable states $s \in S_{e}$ with state changes shown in (15).

$$
\begin{gathered}
\mathcal{E}_{\text {trsf }}=\mathcal{E}_{\text {trsf }}(e, s) \\
\mathcal{S}(e) \leftarrow s
\end{gathered}
$$

The element transporting behavior allows mobile elements to change locations, also affecting nested elements. It is a function of an element which cannot be nested in another element, i.e. $e \in \mathbf{E}: \mathcal{P}(e)=e$ and an allowable location $l \in \mathbf{L}$, with state changes shown in (16).

$$
\begin{aligned}
& \mathcal{E}_{\text {trsp }}=\mathcal{E}_{\text {trsp }}(e, l) \\
& \qquad \mathcal{L}(c) \leftarrow l \forall c: \exists h: \mathcal{P}^{h}(c)=e
\end{aligned}
$$

Finally, although not formally a part of the behavioral framework, two additional events externally manage infrastructure elements. A creation event adds an element (and all nested elements) to the system-of-systems and a destruction event removes an element (and all nested elements) from the system-of-systems. Future work may assign these behaviors to "factory" elements to construct new infrastructure and "decommissioning" elements to remove old infrastructure.

\section{Application Use CAses}

This section presents two application cases to illustrate the applicability of the modeling framework across a range of infrastructure system-of-systems. At a pre-simulation implementation phase, this discussion covers general fit and usefulness of the framework to describe the structure and behavior of infrastructure in the cases of interest. The first case investigates infrastructure supporting future space exploration with high self-sufficiency objectives. The second case investigates sustainable infrastructure investment in Saudi Arabia to meet rapidly growing resource demands.

\section{A. Future Space Exploration Infrastructure}

Future space missions seek to explore distant locations such as near-Earth asteroids (NEAs) and Mars. Underlying the technical challenges of vehicle and mission design there are two key logistical challenges. First, travel to distant locations requires large amounts of propellant due to the physics of rocket propulsion: a round-trip voyage to the surface of Mars using advanced chemical propulsion requires over 200 kilograms of fuel for each kilogram of mass returned to Earth. Second, travel to distant locations takes place over long durations with limited opportunities for resupply, potentially requiring large quantities of contingency resources to be carried along at great expense.

One strategy to improve exploration performance is to close the resource loop and achieve higher self-sufficiency of the remote operations. Advanced life support systems, insitu resource production, and storage depots reduce reliance on resupply but also introduce additional interdependencies. Furthermore, there is active interest in enabling multi-national and commercial enterprises supporting future space exploration [18]. A core principle of the U.S. National Space Policy states that "a robust and competitive commercial space sector is vital to continued progress in space" [19]. In other words, future space exploration planning may involve an infrastructure system-of-systems with strong interdependencies between elements. This case applies the modeling framework to describe structural and behavioral models in the context of a conceptual Martian mission.

1) Structural Models: The nodes of interest include surface regions such as Earth launch sites and Martian exploration sites and stable locations in space such as Earth and Martian orbits. A subset of locations for a Mars exploration application are shown in Fig. 3 with allowable edges corresponding to valid rocket propulsion trajectories. In this case, orbital nodes are represented as time-invariant ellipses rather than point locations. Additional nodes could further disaggregate resource flows, for example one node per surface module to track micro-logistics during exploration operations.

Most resource types in this case are mass-based due to the constraints of rocket propulsion. Main types to consider include several forms of propellant (solid, cryogenic, 


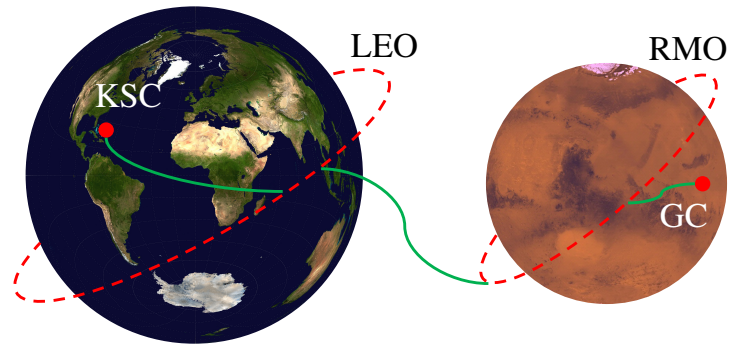

Fig. 3. Subset of locations for Mars exploration including Kennedy Space Center (KSC), Low Earth Orbit (LEO), Reference Mars Orbit (RMO), and Gale Crater (GC) and trajectories KSC-LEO, LEO-RMO, and RMO-GC.

hypergolic, etc.), crew consumables (potable water, oxygen, food), crew waste (solid, liquid, and carbon dioxide), and maintenance items (spare parts). Extensions of the application case may consider electrical energy, human or robotic labor, or information as additional resource types.

Infrastructure elements considered for model instantiations include launch vehicles, in-space vehicles, human and robotic crew, habitats, and in-situ resource production plants. Most elements exist as discrete, mobile infrastructure components managed by various organizations which may be reused across multiple missions in an integrated campaign.

2) Behavioral Models: Resource transforming behaviors are exhibited by crew members through metabolic activity and launch vehicles through propellant consumption. Resource storing behaviors are exhibited by depots and habitats which contain consumables and maintenance items. Some elements such as in-situ resource plants may exhibit resource exchanging behaviors to balance nodal supply and demand, especially across organizational boundaries via financial transactions. Resource transporting behaviors are not largely considered in this scenario due to the large distance scales between nodes, although future extensions such as communications links may use such behaviors.

Element transforming behaviors are exhibited by habitats to represent inhabited and uninhabited states and crew members for nominal and extra-vehicular activity states. Element storing behaviors are exhibited by launch and in-space vehicles which store payload elements and by habitats which store crew members during surface exploration activities. Element transporting behaviors are exhibited by launch and in-space vehicles to traverse between locations to and from the exploration site.

\section{B. Infrastructure Investment in Saudi Arabia}

The Arabian Peninsula has a hot, arid climate which historically limited the expanse of human societies. Since the 1950s the Kingdom of Saudi Arabia has experienced strong economic growth driven in part by use of large natural petroleum reserves. Cities such as Riyadh have grown at an average of $7 \%$ per year from around 50,000 inhabitants in the 1950s to well over 5 million today. At the same time, per capita demands for basic resources such as potable water,

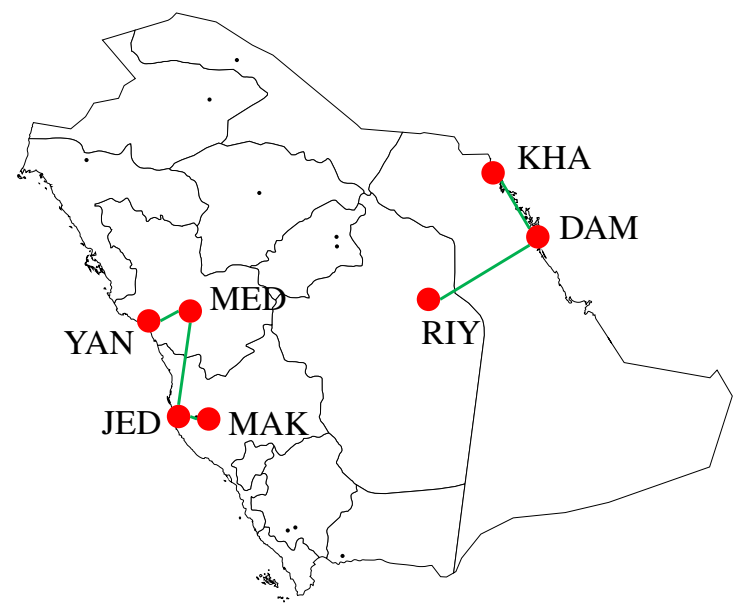

Fig. 4. Subset of locations for Saudi Arabian infrastructure including the cities of Khafji, Dammam, Riyadh, Makkah, Medina, Yanbu, and Jeddah with edges based on physical adjacency.

electricity for cooling, and food also increased as individuals reach higher standards of living.

National development plans seek to invest public funds in new civil infrastructure to meet growing demands; however there are significant challenges to planning. First, there are strong couplings between infrastructure based on geographical, technical, and social factors in Saudi Arabia. For example, Riyadh is positioned at 600 meters in elevation in the center of the peninsula, 400 kilometers from the Persian (Arabian) Gulf and 900 kilometers from the Red Sea. Facing a shortage of potable water from nonrenewable ground reservoirs, its population relies in part on desalinated sea water pumped from the coast, an energy-intensive production and distribution method. Without limited electrical generation and distribution capacity and low domestic petroleum prices, some areas also turn to directly-burning crude which is both environmentallydamaging and reduces export capacity. Finally, historical interest in establishing domestic food security has also relied heavily on rapidly-diminishing natural water aquifers for irrigation in some regions. Second, although Saudi Arabia has a centrally-managed government, national infrastructure planning still involves multiple government ministries and regional administrative divisions acting as a system-of-systems with organizational barriers to collaboration. This case applies the modeling framework to describe the high-level structure and behavior of water-energy infrastructure in Saudi Arabia.

1) Structural Models: The nodes of interest for the context of Saudi Arabia include the major geo-spatial areas and supporting infrastructure regions. The subset of locations focusing on Riyadh shown in Fig. 4 specifically target coastal cities with desalination operations and the interior cities receiving pumped water. The connecting edges represent physically adjacent locations where physical distribution lines exist or may exist.

The resource types of interest include water differentiated by quality (e.g. potable, wastewater, brine, seawater, etc.), 
electrical energy, and other fuels for power plants such as petroleum products. Basic resources could be extended by including monetary resources to illustrate the price and cost of water which is heavily supported by government funds. Additionally, resource types such as carbon dioxide or nonrenewable water or petroleum may be used to illustrate impacts of infrastructure on the natural environment.

Infrastructure elements considered for model instantiations may include zones consisting of people, businesses, and industry, natural and artificial reservoirs, desalination plants, power plants, wastewater treatment plants, and distribution pipelines and networks. Most elements in this case exist as immobile infrastructure components with continuous flows managed by various organizations over time.

2) Behavioral Models: Resource transforming behaviors are exhibited by activities in consumption zones and the various infrastructure plants, for example desalination plants transform electricity and seawater into fresh water. Aggregations of people also can be seen as resource transforming, consuming basic resources to produce societal resources such as income, knowledge, or quality of living. Resource storing behaviors are exhibited by reservoirs and other storage facilities and could also be used to represent natural sources of nonrenewable water or petroleum resources. Resource transporting behaviors are exhibited by the pipelines or other distribution networks which exist on edge locations. Nearly all elements exhibit resource exchanging behaviors to transfer resources to match supply and demand.

Element transforming behaviors are exhibited by societal zones reflecting growth in population or resource consumption and by other infrastructure elements to capture various operational states, for example planned, under construction, operational, and decommissioned. Due to the high level of aggregation, element storing and element transporting behaviors are not exhibited in this application case.

\section{CONCLUSION}

Seeking higher levels of infrastructure systems integration may increase performance and meet sustainability objectives; however it also increases complexity and may lead to poor understanding of interactions and an overall decrease in performance. Especially as infrastructure are managed by independent organizations in a system-of-systems, integrated analysis methods are critical to understanding the cross-system interactions.

This paper contributes a general modeling framework consisting of spatial-structural and temporal-behavioral components at a level suitable for strategic analysis and planning. The spatial-structural components describe a simulation model state while the temporal-behavioral components describe state changes. This formulation builds on existing frameworks which focus on non-executable conceptual frameworks of structure and behavior, aggregated system behavior models independent of spatial structure, and system operation models with static structure for reliability analysis.
Two descriptive application cases illustrate the generality and usefulness of the modeling framework in studying at widely-varying scenarios. The application cases span multiple levels of analysis ranging from individual space system elements supporting human exploration of remote locations to more aggregated infrastructure systems serving entire cities or regions in a modern society.

As a modeling framework, this work is not yet executable as an analysis tool supporting infrastructure systems planning. Future work seeks to implement the modeling framework in a software simulation tool. An implementation would allow the programmatic description of the element behavior models along with context-specific state variables required for each application domain. The two application cases presented are also targeted as application cases for context-specific tools supporting collaborative decision-making for infrastructure system-of-systems.

\section{ACKNOWLEDGMENT}

This work was supported by the Department of Defense (DoD) through a National Defense Science and Engineering Graduate (NDSEG) fellowship with additional support from the Center for Complex Engineering Systems (CCES) at King Abdulaziz City for Science and Technology (KACST) and the Massachusetts Institute of Technology (MIT). The authors would like to thank the numerous colleagues within in the MIT Strategic Engineering group, Jet Propulsion Laboratory, Masdar Institute of Science and Technology, and CCES who have contributed to the development of this modeling framework. In particular, the work of Kaushik Sinha, Thomas Coffee, and Takuto Ishimatsu helped shape this work. The Earth and Mars azimuthal projection source images are courtesy of NASA Visual Earth and NASA/JPL-Caltech and the Saudi Arabia base map is courtesy of d-maps.com, accessed at http://d-maps.com/m/arabie/arabie20.pdf.

\section{REFERENCES}

[1] U.S. Department of Homeland Security, "National infrastructure protection plan," Online, 2009, accessed 1-November 2012. [Online]. Available: http://www.dhs.gov/xlibrary/assets/NIPP_Plan.pdf

[2] United Nations General Assembly, "Resolution A/60/1: 2005 world summit outcome," October 242005.

[3] P. Pederson, D. Dudenhoeffer, S. Hartley, and M. Permann, "Critical infrastructure interdependency modeling: A survey of U.S. and international research," Idaho National Laboratory, Idaho Falls, Idaho, Tech. Rep. INL/EXT-06-11464, August 2006.

[4] T. D. O'Rourke, "Critical infrastructure, interdependencies, and resilience," The Bridge, vol. 37, no. 1, pp. 22-29, Spring 2007.

[5] T. McDaniels, S. Change, K. Peterson, J. Mikawoz, and D. Reed, "Empirical framework for characterizing infrastructure failure interdependencies," Journal of Infrastructure Systems, vol. 13, no. 3, pp. 175184, 2007, doi: 10.1061/(ASCE)1076-0342(2007)13:3(175).

[6] S. M. Rinaldi, J. P. Peerenboom, and T. K. Kelly, "Identifying, understanding, and analyzing critical infrastructure interdependencies," IEEE Control Systems Magazine, pp. 11-25, December 2001.

[7] N. P. Suh, "A theory of complexity, periodicity and the design axioms," Research in Engineering Design, vol. 11, pp. 116-131, 1999.

[8] S. A. Sheard and A. Mostashari, "A complexity typology for systems engineering," in 8th Conference on System Engineering Research, Hoboken, New Jersey, March 2010. 
[9] K. Sinha and O. L. de Weck, "Structural complexity metric for engineered complex systems and its application," in 14th International Design Structure Matrix (DSM) Conference, no. 101, Kyoto, Japan, September 13-14 2012.

[10] M. W. Maier, "Architecting principles for systems-of-systems," Systems Engineering, vol. 1, no. 4, pp. 267-284, 1998.

[11] S. Nader, "Paths to a low-carbon economy-the Masdar example," Energy Procedia, vol. 1, no. 1, pp. 3951-3958, 2009.

[12] National Research Council, Toward Sustainable Critical Infrastructure Systems: Framing the Challenges Workshop Committee. Washington, D.C.: The National Academies Press, 2009.

[13] O. L. de Weck, D. Roos, and C. Magee, Engineering Systems: Meeting Human Needs in a Complex Technological World. MIT Press, January 2012.

[14] B. Bush, L. Dauelsberg, R. LeClaire, D. Powell, S. DeLand, and M. Samsa, "Critical infrastructure protection decision support system (CIP/DSS) project overview," in 2005 International System Dynamics Conference, no. LA-UR-05-1870, Boston, MA, July 17-21 2005.

[15] H.-S. J. Min, W. Beyeler, T. Brown, Y. J. Son, and A. T. Jones, "Toward modeling and simulation of critical national infrastructure interdependencies," IIE Transactions, vol. 39, no. 1, pp. 57-71, 2007.

[16] D. D. Dudenhoeffer, M. R. Permann, and M. Manic, "CIMS: A framework for infrastructure interdependency modeling and analysis," in Proceedings of the 2006 Winter Simulation Conference, L. F. Peronne, F. P. Wieland, J. Liu, B. G. Lawson, D. M. Nichol, and R. M. Fujimoto, Eds., 2006.

[17] P. Zhang and S. Peeta, "A generalized modeling framework to analyze interdependencies among infrastructure systems," Transportation Research Part B, vol. 45, pp. 553-579, 2011.

[18] M. D. Griffin, "Enabling complementary commercial and government enterprises in space," in 62nd International Astronautical Congress, no. IAC-11.E3.4.6. Cape Town, South Africa: International Astronautical Federation, October 3-7 2011.

[19] United States, "National space policy of the United States of America," Online, June 28 2010, accessed 29-August 2012. [Online]. Available: http://www.whitehouse.gov/sites/default/files/national_space policy_6-28-10.pdf 